\title{
Produção, composição e rendimento em queijo do leite de ovelhas Santa Inês tratadas com ocitocina' ${ }^{1}$
}

\section{Louiziane Carvalho Ribeiro ${ }^{2}$, Juan Ramón Olalquiaga Pérez ${ }^{3}$, Pablo Henrique Andrade Carvalho ${ }^{4}$, Fabyano Fonseca e Silva ${ }^{5}$, Joel Augusto Muniz ${ }^{6}$, Gregório Murilo de Oliveira Júnior ${ }^{7}$, Nicholas Vilela de Souza ${ }^{7}$}

\author{
1 Parte da dissertação de Mestrado da primeira autora apresentada à Universidade Federal de Lavras - MG. \\ 2 Mestre em Zootecnia pelo DZO/UFLA. \\ ${ }^{3}$ Departamento de Zootecnia/UFLA. \\ ${ }^{4}$ Graduando em Medicina Veterinária/UFLA \\ ${ }^{5}$ Departamento de Informática/UFV. \\ 6 Departamento de Ciências Exatas/UFLA. \\ 7 Graduando em Zootecnia/UFLA.
}

RESUMO - Objetivou-se avaliar a produção, a composição e o rendimento (na fabricação de queijos) do leite de ovelhas Santa Inês tratadas ou não com ocitocina. Os animais foram mantidos em regime de confinamento e receberam uma dieta com $12 \%$ de PB e $65 \%$ de NDT. As ovelhas foram distribuídas em dois tratamentos: ordenha manual com injeção endovenosa de três UI de ocitocina e ordenha manual sem injeção de ocitocina (grupo controle). Realizou-se uma ordenha diária, de modo que, até a desmama, as ovelhas permaneceram diariamente com os cordeiros durante 14 horas. Os cordeiros foram desmamados aos 60 dias e as ovelhas continuaram sendo ordenhadas uma vez ao dia até o final da lactação. Foram coletadas amostras de leite para análise dos teores de gordura, de sólidos totais e de sólidos desengordurados. A duração média da lactação foi de $225 \pm 27,24$ e 210 $\pm 38,77$ dias para as ovelhas controle e tratadas com ocitocina, respetivamente. Até os 133 dias de lactação, o tratamento com ocitocina $(141,63 \pm 51,52 \mathrm{~kg})$ foi superior ao controle $(89,39 \pm 16,65 \mathrm{~kg})$, entretanto, durante todo o período de lactação, não diferiu $(169,64 \pm 71,05)$ do controle $(119,72 \pm 32,73)$. As porcentagens de gordura $(5,84 \pm 0,44$ e $4,96 \pm 0,47)$, sólidos totais $(17,40 \pm 0,42$ e 16,18 $\pm 0,57)$ e sólidos desengordurados $(11,57 \pm 0,15$ e 11,22 $\pm 0,25)$ no leite foram influenciadas pela aplicação de ocitocina. Os resultados revelaram diferença significativa para as ovelhas tratadas com ocitocina, as quais apresentaram maior produtividade e, inclusive, melhor composição química do leite. A aplicação de ocitocina é recomendada para animais não adaptados à rotina de ordenha. O leite das ovelhas Santa Inês apresentou rendimentos de 4,80; 8,29 e $5,99 \mathrm{~L} / \mathrm{kg}$ para os queijos tipo azeitão, pecorino e roquefort, respectivamente.

Palavras-chave: gordura, hormônio, lactação, ovino

\section{Effects of oxytocin on milk yield and composition of Santa Inês ewes}

ABSTRACT - The objective of this trial was to investigate the effects of oxytocin on milk yield and composition and on cheese yield of Santa Inês sheep receiving a diet containing 12\% CP and 65\% TDN. Animals were maintained in pens and were assigned to one of the following two treatments: hand milking plus an intravenous injection of 3 IU of oxytocin or hand milking without oxytocin injection (control group). Ewes were milked daily and remained with their lambs for a daily period of 14 hours until weaning; lambs were weaned at 60 days of age and ewes still milked once a day until the end of lactation. Milk samples were collected for analysis of fat, total solids, and solids non-fat. Lactation length averaged $225 \pm 27.24$ and $210 \pm 38.77$ for control and oxytocin-treated ewes, respectively. Ewes receiving oxytocin yielded more milk $(141.63 \pm 51.52$ $\mathrm{kg}$ ) than those on the control group $(89.39 \pm 16.65 \mathrm{~kg})$ until 133 days of lactation; however, total milk yield on the entire lactation did not differ significantly comparing oxyitocin-treated $(169.64 \pm 71.05)$ with control $(119.72 \pm 32.73)$ animals Milk contents of fat $(5.84 \pm 0.44$ and $4.96 \pm 0.47)$, total solids $(17.40 \pm 0.42$ and $16.18 \pm 0.57)$ and solids non-fat $(11.57 \pm 0.15$ and 11.22 \pm 0.25 ) differed between both groups of animals, respectively. Results showed improved milk yield and composition on oxytocin-injected ewes; therefore, it is recommended the use of oxytocin to facilitate milk ejection on ewes not adapted to the milking routine. Yields of cheese ( $\mathrm{L}$ of milk/kg of cheese) averaged 4.80, 8.29 and 5.99 $\mathrm{L} / \mathrm{kg}$ for azeitão, pecorino, and roquefort cheeses, respectively.

Key Words: fat, hormone, lactation, sheep 


\section{Introdução}

A atividade leiteira na ovinocultura encontra-se bem estabelecida em diversas regiões do mundo, sobretudo na região Mediterrânea da Europa, em países como França, Itália, Espanha e Grécia, onde a atividade resulta em $66 \%$ da produção mundial de leite de ovelha (Haenlein, 2001). Nesta região, são utilizadas raças especializadas e quase a totalidade do leite produzido é destinada à fabricação de produtos lácteos, principalmente queijos e iogurtes. No Brasil, são poucos os registros de produção de queijos finos como atividade econômica, artesanal ou de subsistência na ovinocultura leiteira.

A composição média do leite de ovelha é de 7,6\% de gordura, 5,6\% de proteína, 19,0\% de sólidos totais, 10,3\% de sólidos desengordurados, 4,7\% de lactose e $4,6 \%$ de caseína (Sevi et al., 2004; Silva, 2003; Nudda et al., 2002; Zamiri et al, 2001; Jandal, 1996, Bencini \& Purvis, 1990). Essa característica lhe confere a capacidade de ser transformado em produtos láteos de alta qualidade com altos rendimentos por litro de leite (Bencini \& Pulina, 1997).

O leite de ovelha, além de alta concentração de sólidos totais (McKusick et al., 2002a), possui níveis elevados de gordura e de caseína, fator importante na elaboração de queijos duros e macios, com características particulares de textura e sabor e com alto valor de mercado (Timperley \& Norman, 1997).

Segundo Furtado (2003), o leite de ovelha apresenta a peculiaridade de não ter caroteno em sua gordura, o que lhe proporciona brancura típica. De acordo com esse autor, a gordura do leite de ovelha, se comparada à do leite de vaca, apresenta maior quantidade de determinados ácidos graxos de cadeia curta, como o capróico (hexanóico), caprílico (octanóico) e cáprico (decanóico).

Jandal (1996), ao estudar os aspectos comparativos dos leites de cabra e ovelha, descreveu que o leite ovino possui maior teor de gordura, sólidos desengordurados, proteína, caseína e cinzas quando comparado ao de cabra. Estas diferenças fazem com que o tempo de coagulação do leite de ovelha seja menor e o coalho mais firme, principalmente em decorrência da quantidade de caseína.

Em pequenos ruminantes, embora a cisterna do úbere possa ser significativa na capacidade de armazenar leite, aproximadamente $75 \%$ da gordura secretada permanece na fração alveolar. Essa gordura é obtida somente sob efeito da ocitocina, hormônio natural secretado pelo animal, responsável pelo reflexo de ejeção do leite e importante para remoção de máxima quantidade de gordura (Labussière, 1988), principalmente em animais não habituados à ordenha.
O reflexo de ejeção do leite é inibido por vários estímulos estressantes (Park \& Jacobson, 1996), sendo importante o adequado manejo de ordenha para maior recuperação do leite rico em sólidos totais.

No Brasil, a criação de ovinos para produção de leite tem se destacado nos últimos anos, principalmente após experiências bem sucedidas de produtores da Serra Gaúcha. Nesta região, a produção e industrialização de leite tiveram início com a raça especializada Lacaune. Entretanto, em virtude dos elevados preços dos animais e das barreiras sanitárias, tornou-se necessário conhecer o potencial leiteiro de raças nativas, como a Santa Inês, especializada em carne, mas com grande vantagem quanto à disponibilidade e adaptação à região.

Neste estudo, avaliaram-se a produção, a composição e o rendimento em queijos do leite de ovelhas Santa Inês tratadas ou não com ocitocina exógena no momento da ordenha.

\section{Material e Métodos}

O experimento foi conduzido no Setor de Ovinocultura da Universidade Federal de Lavras e nas instalações do Laticínio Serrabella Ltda. no período de agosto de 2003 a abril de 2004.

Quatorze ovelhas (3 a 4 anos de idade) de um rebanho de ovelhas Santa Inês em gestação foram selecionadas para estimativa do potencial de produção e análise da composição do leite. Os animais experimentais foram selecionados após o parto, optando-se por fêmeas que haviam parido um único cordeiro. Os animais foram distribuídos aleatoriamente em dois tratamentos: ordenha com injeção de 3 UI de ocitocina (Zamiri et al., 2001), via endovenosa $(n=7)$; e ordenha sem injeção de ocitocina, representando o grupo controle $(n=7)$.

Os animais foram mantidos em regime de confinamento e permaneceram durante todo o tempo em baia coberta. A dieta foi composta de $65 \%$ de cana-de-açúcar (Saccharum officinarum L.), $20 \%$ de polpa cítrica e $15 \%$ de concentrado à base de farelo de algodão (Gossypium hirsuntum), farelo de soja (Glycine max), uréia, fosfato bicálcico, sal comum e suplemento mineral, compondo $12 \%$ de PB e $65 \%$ de NDT na dieta total, formulada para atender às exigências nutricionais de ovelhas em lactação (ARC, 1980). Os animais receberam os alimentos no cocho, na forma de mistura completa, duas vezes ao dia, pela manhã e à tarde, com água e sal mineral à vontade.

Os cordeiros foram mantidos com as mães nos três dias pós-parto e, posteriormente, foram separados todos os dias 
por 10 horas. Durante o tempo em que permaneceram separados, foram amamentados artificialmente com leite de vaca, à vontade (consumo médio de $800 \mathrm{~mL} / \mathrm{animal} / \mathrm{dia})$, durante 60 dias, quando então foram desmamados. Após a ordenha vespertina, os cordeiros foram mantidos com as ovelhas até o dia seguinte, quando foram novamente separados.

Foi realizada uma única ordenha diária, às $17 \mathrm{~h}$, permitindo-se um período de produção de leite de 10 horas. Para ordenha, duas ovelhas por vez foram colocadas em uma plataforma elevada de madeira e presas por canzil. A ordenha foi manual, precedida da injeção de 3 UI de ocitocina na veia epigástrica cranial superficial nos animais tratados.

O leite foi pesado e armazenado a $4^{\circ} \mathrm{C}$ por dois dias, quando foi transportado para o laticínio para avaliação dos processos de fabricação dos queijos.

Aos 60 dias, os cordeiros foram desmamados e as ovelhas continuaram sendo ordenhadas uma vez por dia até o final da lactação, determinado pela produção de menos de $100 \mathrm{~mL}$ de leite/dia (Zamiri et al., 2001; Gootwine \& Goot, 1996; Izadifard \& Zamiri, 1997).

As produções de leite até o desmame, que inicialmente correspondiam ao acúmulo de leite durante 10 horas, foram padronizadas para 24 horas. Foram feitas análises estatísticas para a produção de leite até os 133 dias de lactação (quando a primeira ovelha atingiu produção diária inferior a $100 \mathrm{~mL}$ ) e para a produção total, correspondendo ao leite produzido por cada ovelha ao longo de toda a lactação. A duração da lactação de cada ovelha foi obtida pela soma do total de dias em que as ovelhas foram ordenhadas mais os três dias em que foram mantidas com os cordeiros.

A composição do leite foi avaliada semanalmente até aproximadamente a metade da lactação e quinzenalmente na segunda metade até os 133 dias de lactação. As amostras (200 mL) foram tomadas após homogeneização do leite e armazenadas a $4^{\circ} \mathrm{C}$ até o dia seguinte, quando foram encaminhadas ao laboratório do laticínio.

Foram analisados os teores de gordura, sólidos totais (ST) e sólidos totais desengordurados (STD) do leite. Todas as análises foram realizadas em triplicata. $\mathrm{O}$ teor de gordura foi determinado pelo método butirométrico de Gerber e o de sólidos totais (ST), por meio da fórmula de Fleishemam, em que $\mathrm{G}$ corresponde à porcentagem de gordura da amostra e D à sua densidade: $\mathrm{ST}=1,2 * \mathrm{G}+(2,665$ * (100* D - 100) / D). Para encontrar o valor da densidade, adotou-se o método do termolactodensímetro. O valor de sólidos totais desengordurados (STD) foi obtido pela diferença entre a porcentagem de sólidos totais e a de gordura da amostra (Abreu, 1999).
Foram feitos estudos sobre a técnica de fabricação de queijos dos tipos azeitão, pecorino e roquefort, efetuando-se análise do rendimento final des ses queijos. Os estudos foram desenvolvidos considerando-se o total de leite dos dois tratamentos, não havendo comparação entre tratamentos.

O período de maturação variou de acordo com o tipo de queijo fabricado: o roquefort teve maturação de até três meses, o azeitão de 30 dias e opecorino de seis meses.

A análise de variância usada para avaliar a influência dos tratamentos sobre as variáveis dia da produção máxima, produção máxima, produção de leite até 133 dias de lactação, média da produção até os 133 dias, produção de leite total, média da produção total, duração da lactação, teor de gordura $(\%)$, teor de sólidos totais $(\%)$, teor de sólidos totais desengordurados $(\%)$, produção de gordura $(\mathrm{kg})$, produção de sólidos totais $(\mathrm{kg})$ e produção de sólidos totais desengordurados $(\mathrm{kg})$ foi realizada por meio do procedimento GLM do SAS $^{\circledR}$ (SAS, 1990), utilizando-se o modelo a seguir:

$$
Y_{i j}=\mu+T_{i}+\varepsilon_{i j}
$$

em que: $Y_{i j}=$ valor da variável avaliada no tratamento $i$ na repetição $\mathrm{j}(\mathrm{j}=1,2,3, \ldots, 7) ; \mu=$ média geral; $\mathrm{T}_{\mathrm{i}}=$ efeito do tratamento $\mathrm{i}(\mathrm{i}=1,2)$; e $\varepsilon_{\mathrm{ij}}=$ erro associado a cada observação, considerado aleatório, independente, com distribuição normal, média zero e variância $\sigma^{2}$.

\section{Resultados e Discussão}

As médias obtidas para as variáveis dia da produção máxima, produção diária máxima, produção até 133 dias de lactação, média da produção até 133 dias de lactação, produção total, média da produção total e duração da lactação, para cada tratamento, são apresentadas na Tabela 1.

O pico de produção de leite ocorreu, em média, no $37 \underline{0}$ e 350 dias de lactação, respectivamente, para as ovelhas controle e tratadas com ocitocina. Estes resultados foram próximos aos observados por Cardellino \& Benson (2002), que afirmaram que o pico de produção de leite ocorreu entre o 210 e o 30 o dia de lactação. A produção máxima das ovelhas tratadas com ocitocina foi, em média, 1,7 vez maior $(\mathrm{P}<0,01)$ que a do grupo controle.

O total de leite produzido até os 133 dias de lactação foi de $89,39 \mathrm{~kg}$ para as ovelhas controle e de $141,63 \mathrm{~kg}$ para aquelas tratadas com ocitocina $(\mathrm{P}<0,05)$. Segundo Kremer et al. (1996), a produção de leite de ovelhas Corriedale, uma raça que também não é especializada, foi de $90 \mathrm{~L}$ em 120 dias de lactação, inferior à observada neste estudo com a aplicação de ocitocina. A produção diária de leite até os 133 dias 
Tabela 1 - Produção de leite e duração da lactação de ovelhas Santa Inês

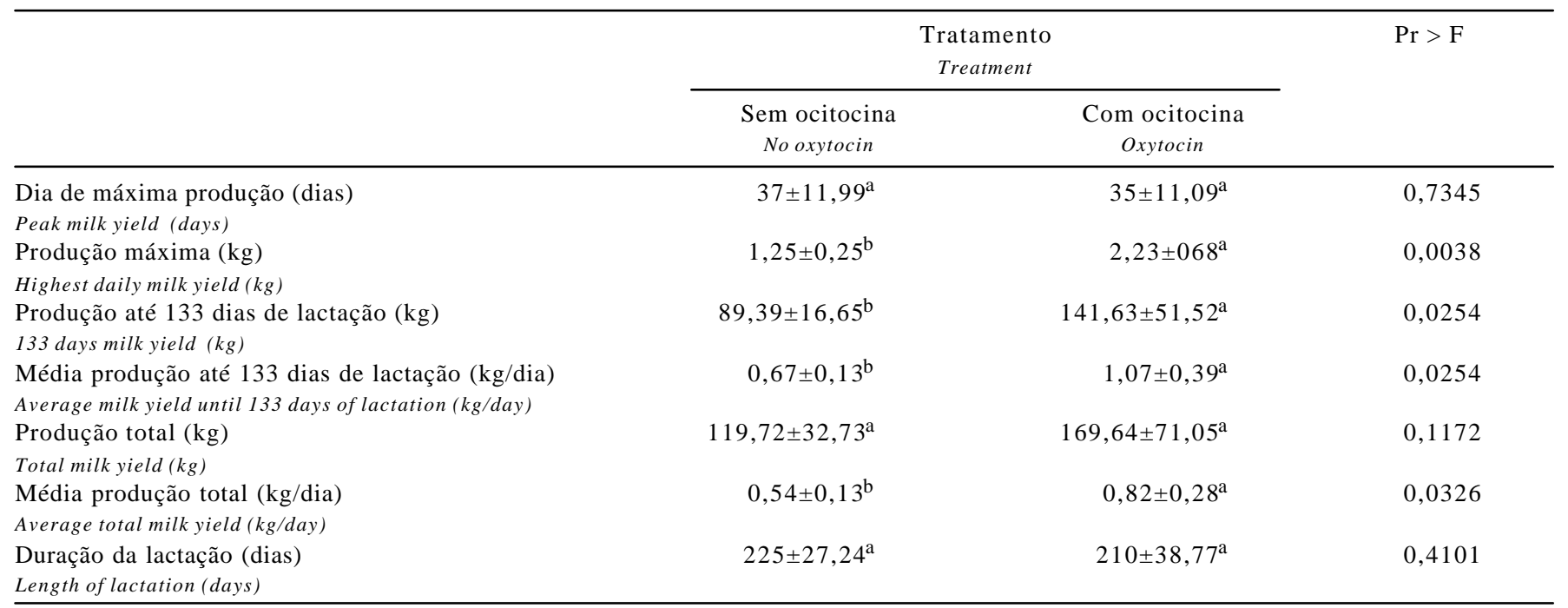

* Médias com letras diferentes nas linhas diferem $(P<0,05)$ pelo teste $F$.

${ }^{*}$ Means within rows with different superscripts differ $(P<0.05)$ by $F$ test.

de lactação foi maior $(\mathrm{P}<0,05)$ nas ovelhas tratadas com ocitocina. Os valores encontrados neste estudo, principalmente nas ovelhas tratadas com ocitocina $(1,07 \mathrm{~kg})$, foram próximos aos observados por Ugarte et al. (2001) em ovelhas especializadas Lacaune, Awassi e Assaf, que apresentaram produções diárias de 1,$9 ; 1,5$ e 2,0 L, respectivamente. A menor produção de leite das ovelhas do grupo controle pode ser atribuída à falha no reflexo de ejeção do leite, pois, como foram ordenhadas pela primeira vez, o manejo de ordenha causou-lhes estresse, prejudicando a ação da ocitocina endógena sobre as células mioepiteliais dos alvéolos. Conseqüentemente, a fração alveolar do leite ficou retida no úbere, resultando em menor produção. Similarmente, Bencini et al. (1992) concluíram que a aplicação de ocitocina aumentou a quantidade de leite extraído ( $\mathrm{P}<0,01)$. Segundo Zamiri et al. (2001), o efeito galactopoético da ocitocina pode estar relacionado ao aumento na taxa de secreção de leite. Duas hipóteses têm sido propostas: a primeira refere-se à influência da ocitocina na eficiência de remoção de leite, que aumenta a secreção pela redução do efeito inibitório da lactação (Wilde \& Peaker, 1990); a segunda hipótese atribui à ocitocina influência direta na produtividade ou manutenção das células epiteliais mamárias (Ballou et al., 1993).

A duração da lactação das ovelhas do grupo controle e daquelas tratadas com ocitocina foi de 225 e 210 dias, respectivamente $(\mathrm{P}>0,05)$, observando-se que a duração média da lactação neste estudo foi superior à observada por Gootwine \& Goot (1996) nas raças especializadas Awassi (200 dias) e East Friesian (186 dias).
A produção total de leite durante toda lactação foi de $119,72 \mathrm{~kg}$ para as ovelhas controle e de 169,64 kg para as ovelhas que receberam ocitocina $(\mathrm{P}>0,05)$. Estes valores foram próximos aos obtidos com raças especializadas, como Lacaune, Awassi e East Friesian, de 270, 247 e 161 L de leite, com duração de lactação de 165, 200 e 186 dias, respectivamente (Barillet et al., 2001; Gootwine \& Goot, 1996). Quando considerada a produção total de leite durante todo o período de lactação, não foi observada diferença entre os tratamentos $(\mathrm{P}>0,05)$. Este resultado pode ser explicado, em parte, pelas variações de produção entre os animais, ocasionadas pela falta de padronização da raça, que não foi submetida à seleção para esta característica. No entanto, como o período de lactação variou entre os animais (225 dias para as ovelhas controle e 210 dias para aquelas tratadas com ocitocina), quando calculada a média de produção diária, houve diferença $(\mathrm{P}<0,05)$ entre os tratamentos, com valores de 0,54 e $0,84 \mathrm{~kg}$, respectivamente.

As médias das variáveis teor de gordura, produção de gordura, teor de sólidos totais, produção de sólidos totais, teor de sólidos totais desengordurados e produção de sólidos totais desengordurados obtidas em cada tratamento são demonstradas na Tabela 2.

O teor de gordura do leite das ovelhas tratadas com ocitocina foi de $5,84 \%$ ( $8,32 \mathrm{~kg}$ de gordura), enquanto, no leite das ovelhas do grupo controle, foi de 4,96\% (4,45 kg) $(\mathrm{P}<0,01)$. Bencini et al. (1992), no entanto, relataram que diferentes doses de ocitocina não afetaram o conteúdo de gordura do leite.Zamiri et al. (2001) observaram que ovelhas tratadas com ocitocina apresentaram maior teor de gordura 
Tabela 2 - Produção e composição do leite de ovelhas Santa Inês

Table 2 - Milk yield and composition on Santa Inês ewes injected or not with oxytocin

\begin{tabular}{|c|c|c|c|}
\hline & \multicolumn{2}{|c|}{$\begin{array}{c}\text { Tratamento } \\
\text { Treatment }\end{array}$} & \multirow[t]{2}{*}{$\operatorname{Pr}>\mathrm{F}$} \\
\hline & $\begin{array}{l}\text { Sem ocitocina } \\
\text { No oxytocin }\end{array}$ & $\begin{array}{c}\text { Com ocitocina } \\
\text { Oxytocin }\end{array}$ & \\
\hline Teor de gordura (\%) (Milk fat, \%) & $4,96 \pm 0,47^{\mathrm{b}}$ & $5,84 \pm 0,44^{\mathrm{a}}$ & 0,0038 \\
\hline Produção de gordura $(\mathrm{kg})$ (Milk fat yield, $k g$ ) & $4,45 \pm 0,98^{\mathrm{b}}$ & $8,32 \pm 3,19^{\mathrm{a}}$ & 0,0097 \\
\hline Teor de sólidos totais (\%) (Milk total solids, \%) & $16,18 \pm 0,57^{b}$ & $17,40 \pm 0,42^{\mathrm{a}}$ & 0,0006 \\
\hline Produção de sólidos desengordurados ( $\mathrm{kg}$ ) (Milk solids non-fat yield, $\mathrm{kg}$ ) & $10,05 \pm 2,04^{b}$ & $16,38 \pm 5,91^{\mathrm{a}}$ & 0,0202 \\
\hline
\end{tabular}

${ }^{*}$ Médias com letras diferentes nas linhas diferem $(P<0,05)$ pelo teste $F$.

* Means within rows with different superscripts differ $(P<0.05)$ by $F$ test.

no leite, resultado semelhante aos encontrado neste estudo, em que o uso de ocitocina exógena promoveu aumento na porcentagem e na produção de gordura do leite. As ovelhas não tratadas com ocitocina, provavelmente por não estarem adaptadas à rotina de ordenha, apresentaram falha no reflexo de ejeção do leite e retiveram no úbere a fração de leite alveolar, rica em gordura (Labussière et al., 1969). Segundo Park \& Jacobson (1996), o estresse aumenta a descarga de catecolaminas que inibem a ação da ocitocina endógena sobre as células mioepiteliais dos alvéolos. De acordo com McKusick et al. (2002a), a atividade mediada pela ocitocina na contração das células mioepiteliais é essencial para remoção da gordura do leite do úbere.

O teor de sólidos totais do leite das ovelhas não tratadas e daquelas tratadas com ocitocina até os 133 dias de lactação foram de 16,18 e 17,40\%, respectivamente $(\mathrm{P}<0,01)$. A quantidade de sólidos totais produzida nesse período também foi maior $(\mathrm{P}<0,05)$ nas ovelhas tratadas com ocitocina, o que comprova a importância da aplicação de ocitocina no aumento de sólidos totais no leite. De acordo com McKusick et al. (2002b), a ejeção do leite em ovelhas leiteiras é essencial para recuperação do leite rico em sólidos totais.

O uso de ocitocina no momento da ordenha aumentou a porcentagem $(\mathrm{P}<0,01)$ e quantidade $(\mathrm{P}<0,05)$ de sólidos totais desengordurados do leite produzido até os 133 dias de lactação.

O teor de gordura leite de ovelha varia de 4,00 a 9,00\%, o de teor de sólidos totais de 16,7 a $19,70 \%$ e o de sólidos totais desengordurados de 10,33 a 12,68\% (Ochoa-Cordero et al., 2002; Jandal, 1996; Kremer et al., 1996; Simos et al., 1996; Hassan, 1995; Boujenane \& Lairini, 1992; Peeters et al., 1992; Bencini \& Purvis, 1990; Mann, 1988). As porcentagens de gordura, sólidos totais e de sólidos desengordurados observada neste estudo estão dentro do intervalo descrito na literatura. Contudo, ao comparar a porcentagem de gordura do leite encontrada àquelas observadas em raças especializadas, nota-seque os valoresobtidos neste estudo são inferiores. Animais de raças especializadas, como Lacaune, Sarda, East Friesian e Awassi, produzem leite com média de 7,68;6,72;6,17 e 6,67\% de gordura, respectivamente (McKusick et al., 2002a b; Nuda et al., 2002; Barillet et al., 2001; Bencini \& Pulina, 1997; Casoli et al., 1989). Ressalta-se, no entanto, que essas raças passaram por intenso processo de seleção genética.

Como demonstrado na Tabela 3 , o rendimento mensurado para o queijo tipo azeitão após 30 dias de maturação foi de $4,80 \mathrm{~L}$ de leite/kg de queijo produzido. Esse resultado foi próximo ao encontrado por Soryal (2002), de 4,63 L/kg de queijo em período de maturação também mais curto.

$\mathrm{O}$ rendimento final do queijo tipopecorino após seis meses de maturação foi de $8,29 \mathrm{~L}$ de leite/kg de queijo produzido. De acordo com Michelon (2005), o rendimento desse tipo de queijo é de $5 \mathrm{~L}$ de leite/kg de queijo produzido, observando-se que, após nove meses de maturação, o queijo perde $30 \%$ de seu peso. O rendimento encontrado neste estudo para o queijo tipo pecorino foi menor, pois as pesagens dos queijos foram realizadas após o período de maturação, quando ocorre perda de peso do queijo. Moura (1997), ao estudar o rendimento do queijo parmesão, similar ao pecorino, porém feito com leite de vaca, observou, após a retirada da prensa, rendimentos de 9,16 a 9,68 L de leite/kg de queijo produzido.

Tabela 3 - Rendimento em queijo do leite de ovelhas Santa Inês Table 3 - Cheese yield on Santa Inês ewes

\begin{tabular}{lccc}
\hline $\begin{array}{l}\text { Queijo } \\
\text { Cheese }\end{array}$ & $\begin{array}{c}\text { Leite }(\mathrm{L}) \\
\text { Milk }(L)\end{array}$ & $\begin{array}{c}\text { Queijo }(\mathrm{kg}) \\
\text { Cheese }(\mathrm{kg})\end{array}$ & $\begin{array}{c}\text { Rendimento }(\mathrm{L} / \mathrm{kg}) \\
\text { Yield }(\mathrm{L} / \mathrm{kg})\end{array}$ \\
\hline Tipo azeitão & 35,00 & 7,29 & 4,80 \\
Tipo pecorino & 17,00 & 2,05 & 8,29 \\
Tipo roquefort & 55,5 & 9,27 & 5,99 \\
\hline
\end{tabular}


Após três meses de maturação, o rendimento final do queijo tipo roquefort foi de $5,99 \mathrm{~L}$ de leite $/ \mathrm{kg}$ de queijo produzido. De acordo com Michelon (2005), o rendimento desse tipo de queijo é de $4,5 \mathrm{~L}$ de leite/ $\mathrm{kg}$ de queijo produzido e, após seis meses de maturação, ocorre perda de $10 \%$ de seu peso. O rendimento do queijo gorgonzola (similar ao roquefort) encontrado por Paciulli (2001) variou de 7,29 a 7,72 L de leite/kg de queijo produzido.

\section{Conclusões}

As ovelhas Santa Inês utilizadas neste estudo, mesmo que de uma raça tradicionalmente explorada para produção de carne, apresentaram alta produção de leite e duração da lactação elevada. O leite produzido apresentou composição química satisfatória e bom rendimento para fabricação de queijos finos.

O uso de ocitocina exógena no momento da ordenha interferiu na quantidade e na qualidade do leite produzido e não influenciou a persistência da lactação, sendo recomendado para animais não adaptados à rotina de ordenha.

\section{Literatura Citada}

ABREU, L.R. Tecnologia de leite e derivados. Lavras: Universidade Federal de Lavras, 1999. 215p.

AGRICULTURAL RESEARCH COUNCIL - ARC. The nutrient requirement of farm animals. London: ARC, 1980. 351p.

BALLOU, L.U.; BLECK, J.L.; BLECK, G.T. et al. The effects of daily oxytocin injections before and after milking on milk production, milk plasmin, and milk composition. Journal of Dairy Science, v.76, n.6, p.1544-1549, 1993

BARILlET, F.; MARIE, C.; JACQUIN, M. et al. The French Lacaune dairy sheep breed: use in France and abroad in the last 40 years. Livestock Production Science, v.71, n.1, p.17-29, 2001.

BENCINI, R.; MARTIN, G.B.; PURVIS, I.W. et al. Use of oxytocin to measure milk output in Merino ewew and its effect on fat content. Australian Journal of Experimental Agriculture, v.32, n.5, p.601-603, 1992.

BENCINI, R.; PULINA, G. The quality of sheep milk: a review. Australian Journal of Experimental Agriculture, v.37, n.5, p.485-504, 1997.

BENCINI, R.; PURVIS, I.W. The yield and composition of milk from Merino sheep. Proceedings of the Australian Society of Animal Production, v.18, p.144-147, 1990.

BOUJENANE, I.; LAIRINI, K. Genetic and environmental effects on milk production and fat percentage in D'man and Sardi ewes and their crosses. Small Ruminant Research, v. 8, n.3, p.207215,1992

CARDELLINO, R.A.; BENSON, M.E. Lactation curves of commercial ewes rearing lambs. Journal of Animal Science, v.80, n.1, p.23-27, 2002

CASOLI, C.; DURANTI, E.; MORBIDINI, L. et al. Quantitative and compositional variations of Massese sheep milk by parity and stage of lactation. Small Ruminant Research, v.2, n.1, p.47-62, 1989
FURTADO, M.M. Queijos finos maturados por fungos. São Paulo: Milkbizz, 2003. 128p.

GOOTWINE, E.; GOOT, H. Lamb and milk production of Awassi and East-Friesian sheep and their crosses under Mediterranean environment. Small RuminantResearch, v.20, n.3, p.255260, 1996

HAENLEIN, G.F.W. Past, present, and future perspectives of small ruminant dairy research. Journal of Dairy Science, v.84, n.9, p.2097-2115, 2001

HASSAN, H.A. Effects of crossing and environmental factors on production and some constituents of milk in Ossimi and Saidi sheep and their crosses with Chios. Small Ruminant Research, v.18, n.2, p.165-172, 1995.

IZADIFARD, J.; ZAMIRI, M.J. Lactation performance of two Iranian fat-tailed sheep breeds. Small Ruminant Research, v.24, n.2, p.69-76, 1997.

JANDAL, J.M. Comparative aspects of goat and sheep milk. Small Ruminant Research, v.22, n.2, p.177-185, 1996.

KREMER, R.; ROSÉS, L.; RISTA, L. et al. Machine milk yield and composition of non-dairy Corriedale sheep in Uruguay. Small Ruminant Research, v.19, n.1, p.9-14, 1996.

LABUSSIÈRE, J. Review of physiological and anatomical factors influencing the milking ability of ewes and the organization of milking. Livestock Production Science, v.18, n.3-4, p.253-274, 1988.

LABUSSIÈRE, J.; MARTINET, J.; DENAMUR, R. The influence of the milk ejection reflex on the flow rate during the milking of ewes. Journal of Dairy Research, v.36, n.2, p.191-201, 1969.

MANN, E.J. Ewes' and goats' milk and products: part 1. Dairy Industries International, v.53, n.3, p.11-12, 1988.

McKUSICK, B.C.; THOMAS, D.L.; BERGER, Y.M. et al. Effect of milking interval on alveolar versus cisternal milk accumulation and milk production and composition in dairy ewes. Journal of Dairy Science, v.85, n.9, p.2197-2206, 2002a.

McKUSICK, B.C.; WILTBANK, M.C.; SARTORI, R. et al. Effect of presence or absence of corpora lutea on milk production in East Friesian dairy ewes. Journal of Dairy Science, v.85, n.4, p.790-796, 2002b.

MICHELON, T. Casa da ovelha. [2005]. Disponível em: <http:/ /www.casadaovelha.com.br/> Acesso em: 30/04/2005.

MOURA, C.J. Efeito do resfriamento do leite sobre o rendimento e lipólise do queijo tipo parmesão. Lavras: Universidade Federal de Lavras, 1997. 77p. Dissertação (Mestrado em Ciência dos Alimentos) - Universidade Federal de Lavras.

NUDDA, A.; BENCINI, R.; MIJATOVIC, S. et al. The yield and composition of milk in Sarda, Awassi, and Merino sheep milked unilaterally at different frequencies. Journal of Dairy Science, v.85, n.11, p.2879-2884, 2002.

OCHOA-CORDERO, M.A.; TORRES-HERNÁNDEZ, G.; OCHOAALFARO, A.E. et al. Milk yield and composition of Rambouillet ewes under intensive management. Small Ruminant Research v.43, n.3, p.269-274, 2002.

PACIULLI, S.O.D.; ABREU, L.R.; PINTO, S.M. et al. Análise físicoquímica do leite pasteurizado pelos sistemas HTST e ejetor de vapor e do queijo tipo gorgonzola elaborado com estes leites. Revista do Instituto de Laticínios Cândido Tostes, v.56, n. 318, p.21-30, 2001.

PARK, C.S.; JACOBSON, N.L. Glândula mamária e lactação. In: SWENSON, M.J.; REECI, W.O. (Eds.) Dukes: fisiologia dos animais domésticos. 11.ed. Rio de Janeiro: Guanabara Koogan, 1996. p.645-659.

PEETERS, R.; BUYS, N.; ROBIJNS, L. et al. Milk yield and milk composition of Flemish Milksheep, Suffolk and Texel ewes and their crossbreds. Small Ruminant Research, v.7, n.4, p.279288, 1992.

SATATISTICAL ANALYSIS SYSTEM - SAS. SAS/IML software usage and refernce. version 6. Cary: 1990. 501p. 
SEVI, A.; ALBENZIO, M.; MARINO, R. et al. Effects of lambing season and stage of lactation on ewe milk quality. Small Ruminant Research, v.51, n.3, p.251-259, 2004.

SILVA, M.G.C.M. Produção de caprinos. Lavras: UFLA/FAEPE, 2003. 56p

SIMOS, E.N.; NIKOLAOU, E.M.; ZOIOPOULOS, P.E. Yield, composition and certain physicochemical characteristics of milk of the Epirus mountain sheep breed. Small Ruminant Research, v.20, n.1, p.67-74, 1996.

SORYAL, K.A. Thirst and ewe's milk processing. Small Ruminant Research, v.45, n.2, p.95-99, 2002.

TIMPERLEY, C.; NORMAN, C. O livro de queijos. São Paulo: Manole, 1997. 119p.
UGARTE, E.; RUIZ, R.; GABIÑA, D. et al. Impact of high-yielding foreign breeds on the Spanish dairy sheep industry. Livestock Production Science, v.71, n.1, p.3-10, 2001.

WILDE, C.J.; PEAKER, M. Review: autocrine control in milk secretion. Journal of Agricultural Science, v.114, n.3, p.235-238, 1990.

ZAMIRI, M.J.; QOTBI, A.; IZADIFARD, J. Effect of daily oxytocin injection on milk yield and lactation length in sheep. Small Ruminant Research, v.40, n.2, p.179-185, 2001.

Recebido: 07/07/05 Aprovado: 28/09/06 